\title{
Diagnostic Accuracy of Echo-planar Diffusion-weighted Imaging in the Diagnosis of Intra-cerebral Abscess by Taking Histopathological Findings as the Gold Standard
}

\author{
Hina Siddiqui $^{1}$, Sameera Vakil ${ }^{1}$, Maria Hassan ${ }^{2}$ \\ 1. Radiology, Dow University of Health Sciences (DUHS), Karachi, PAK 2. Radiology, Dr. Ziauddin \\ Hospital, Karachi, PAK
}

$\square$ Corresponding author: Hina Siddiqui, hinaa1085@gmail.com

Disclosures can be found in Additional Information at the end of the article

\section{Abstract}

\section{Objective}

To determine the diagnostic accuracy of echo-planar diffusion-weighted imaging (DWI) in the diagnosis of intra-cerebral abscesses by taking histopathological findings as the gold standard.

\section{Subject and methods}

A retrospective cross-sectional study was performed from July 2014 to June 2015 at a tertiary care hospital in Karachi. A total of 462 patients, who were referred for magnetic resonance imaging (MRI) brain, presenting with clinical suspicion of an intra-cerebral abscess on the basis of clinical signs and symptoms, were included in this study. MR imaging was performed. All patients subsequently underwent surgery. The histopathological findings of these patients were collected and compared with echo-planar diffusion-weighted MRI findings. A proforma was used to record the findings.

\section{Results}

The mean age of the patients was $47.39 \pm 13.54$ years. The sensitivity, specificity, positive predictive value, negative predictive value, and diagnostic accuracy of echo-planar diffusionweighted MRI in the diagnosis of intra-cerebral abscesses was 85.64\%, 95.88\%, 93.82\%, 90.14\%, and $91.56 \%$, respectively.

Received 05/07/2019

Review began 05/12/2019 Review ended 05/13/2019 Published 05/16/2019

(C) Copyright 2019

Siddiqui et al. This is an open access article distributed under the terms of the Creative Commons Attribution License CC-BY 3.0., which permits unrestricted use, distribution, and reproduction in any medium, provided the original author and source are credited.

\section{Conclusion}

Brain abscesses and necrotic tumors are, most of the time, difficult to differentiate on routine conventional imaging, and prompt diagnosis is important, as an untreated brain abscess could be lethal. Diffusion imaging can aid in the diagnosis and further management plan so as to help in improved patient care. Although this sequence has high sensitivity and specificity, it should be used in addition to conventional imaging and not as a replacement of histopathology.

Categories: Radiology

Keywords: intra-cerebral abscess, echo-planar diffusion-weighted imaging 


\section{Introduction}

An intra-cerebral abscess is a significant healthcare problem in the developing world due to large-scale poverty, illiteracy, and lack of hygiene [1-2]. It starts as a localized area of cerebritis, which is subsequently converted into a collection of pus within a well-vascularized capsule [2]. Predisposing conditions are present in up to $86 \%$ of patients and mostly consist of contiguous or distant foci of infection [3]. Intra-cerebral abscesses constitute up to $8 \%$ of the intracranial space-occupying lesions seen in the developing countries [4]. It is most common in the second to third decades of life, with a male to female ratio of $3.4: 1$ and a mortality rate of about $11.3 \%$ [5]. Its prevalence is anticipated to be $2 \%-14 \%$ [6].

An intra-cerebral infection is a true neurosurgical emergency that requires rapid diagnosis and appropriate management, as its clinical course may become fulminant [1]. Advances in surgery, neuroimaging, and antibiotics have significantly improved the outcomes, but mortality and morbidity remain high [1-2]. Computed tomography (CT) scans and magnetic resonance imaging (MRI) are the main noninvasive modalities used to diagnose intra-cerebral abscesses [4]. However, the imaging features of abscesses are nonspecific and may simulate those of cystic rim-enhancing tumors. Because of this limited ability of conventional CT and MRI, new and more advanced techniques are urgently needed [4,7].

Diffusion-weighted (DW) MRI using the echo-planar technique has been shown to be useful for distinguishing brain abscesses from necrotic or cystic brain tumors [8-9]. DW MRI characterizes a lesion based on the free diffusing property of water molecules in that lesion [10-11]. An intracerebral abscess causes diffusion restriction due to inflammatory cells, necrotic tissue, and proteins in the pus, which returns bright signals on DW MRI $[4,10]$. This feature differentiates it from necrotic tumors, which are usually not diffusion restricted [10]. This carries significant implications on the intent and nature of treatment [9]. Reddy et al. reported the sensitivity and specificity of echo-planar diffusion-weighted imaging (DWI) for detecting intra-cerebral abscesses as $96 \%$ and $96 \%$, respectively [9].

This study aims to determine the diagnostic accuracy of echo-planar diffusion-weighted MRI in the diagnosis of intra-cerebral abscesses. If this study shows high sensitivity and specificity of echo planar DWI, then early diagnosis would eliminate the need for invasive diagnostic procedures, lead to early commencement of treatment, and, hence, will substantially reduce the morbidity of this condition. There are only a few studies on this topic in our population with relatively small sample size. In this study, we included 462 subjects, which gave stronger results.

\section{Materials And Methods}

A retrospective cross-sectional study performed for one year from July 2014 to June 2015 at a tertiary care hospital in Karachi. Non-probability, consecutive sampling was used. Informed consent was waived, as it was a retrospective study. A total of 462 patients of ages 18 to $70 \mathrm{yrs}$ (mean age $47.39 \pm 13.54$ years), both genders, who were referred for MRI brain, presenting with clinical suspicion of an intra-cerebral abscess on the basis of clinical signs and symptoms, as described below:

- The clinical triad of moderate to severe headache (4-10 on the visual assessment scale), fever $(>101 \mathrm{~F})$, and altered mental status (assessed by Glasgow Coma Scale $(\mathrm{GCS}) \leqslant 15$ ) of one week or more in duration.

- Other associated features like generalized seizures (assessed clinically by jerky movements), neck stiffness, blurring of vision, vomiting (at least two episodes per day for at least one day), and limb weakness assessed by clinical examination. 


\section{Cureus}

The exclusion criteria included:

- Patients who do not undergo surgery or are lost to follow-up

- Previously diagnosed cases of an intra-cerebral abscess or brain tumor

- Patients in whom no ring-enhancing lesion is seen on brain MRI

- Previous history of radiotherapy, chemotherapy, or surgery

MR imaging was performed on a 1.5 Tesla system (Atlas, Toshiba, Japan). The protocol consists of T1-weighted spin-echo (SE) images (TR/TE: 615/12 ms), T2-weighted turbo SE (TR/TE: 4000/98 ms), fluid-attenuated inversion-recovery sequences (TR/TE: 9000/119 ms), and postcontrast T1- weighted images. Diffusion-weighted imaging was obtained in the axial plane using an echo-planar single-shot sequence with the following parameters: TR/TE 4500/83 ms; matrix size, 256× 256; FOV, $220 \mathrm{~mm}$; slice thickness, $5 \mathrm{~mm}$. The MRI was interpreted by a senior radiologist having more than seven years of experience.

All patients subsequently underwent surgery. The histopathological findings of these patients were collected and compared with the echo-planar diffusion-weighted MRI findings. A proforma was used to record the findings.

Statistical data analysis was done using the Statistical Package for the Social Sciences (SPSS version 20; IBM Corporation, Armonk, NY, US). The age and size of the abscess were presented by the mean and standard deviation. Frequency and percentage were computed for gender. A $2 \times 2$ table was constructed and the sensitivity, specificity, positive predictive value (PPV), negative predictive value (NPV), and diagnostic accuracy of echo-planar diffusion-weighted MRI for the diagnosis of an intra-cerebral abscess were calculated by taking histopathological findings as the gold standard. Kappa statistics were calculated to see the strength of the association between the two modalities. $\mathrm{K}>0.8$ was taken as significant. Stratification with respect to the age and size of the abscess was done. Post-stratification kappa statistics were also calculated.

\section{Results}

A total of 462 patients, who were referred for MRI brain, presenting with clinical suspicion of an intra-cerebral abscess on the basis of clinical signs and symptoms, were included in this study. One of the patients, age 70 years, showed a well-defined abnormal signal intensity area in the right hemipons on $\mathrm{T} 2 \mathrm{~W}$ and $\mathrm{T} 1 \mathrm{~W}$ images, with diffusion restriction and peripheral postcontrast enhancement suggestive of an abscess (Figures 1-2).

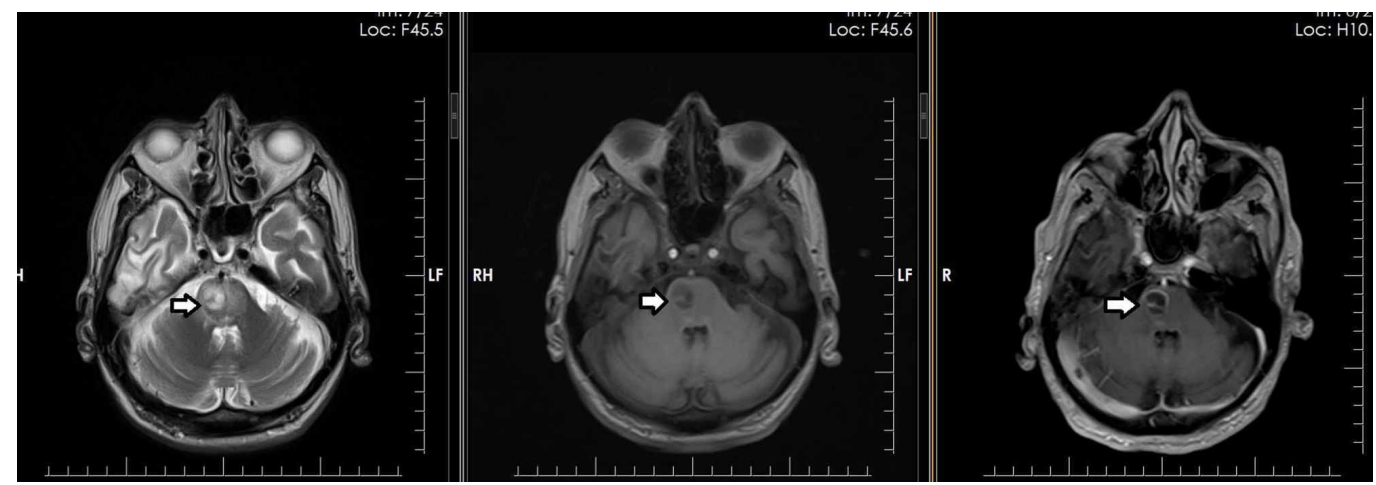

FIGURE 1: Seventy-year-old patient with a right hemipons 


\section{Cureus}

\section{abscess showing peripheral enhancement on post-contrast}

images

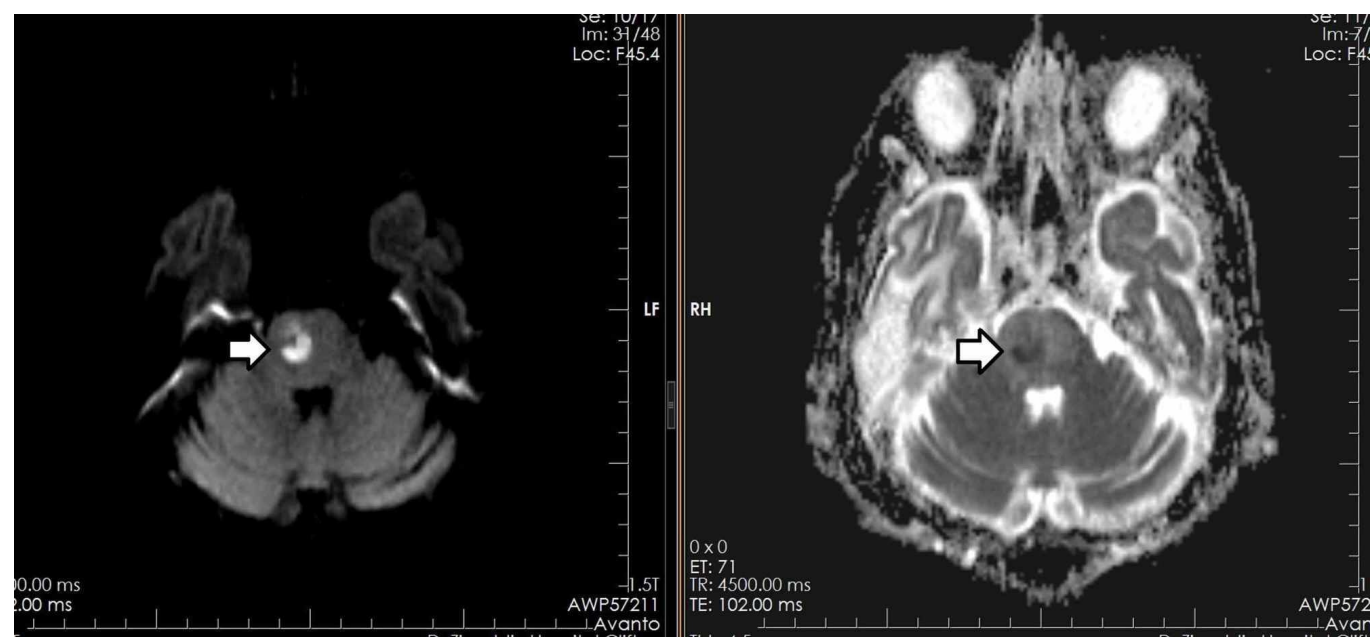

FIGURE 2: Similar patient with a right hemipons abscess showing diffusion restriction on DWI and ADC sequences

DWI: diffusion-weighted magnetic resonance imaging; ADC: apparent diffusion coefficient

Regarding the demographics of the patient, it was observed that patients above the age of 40 years were more affected (Figure 3).

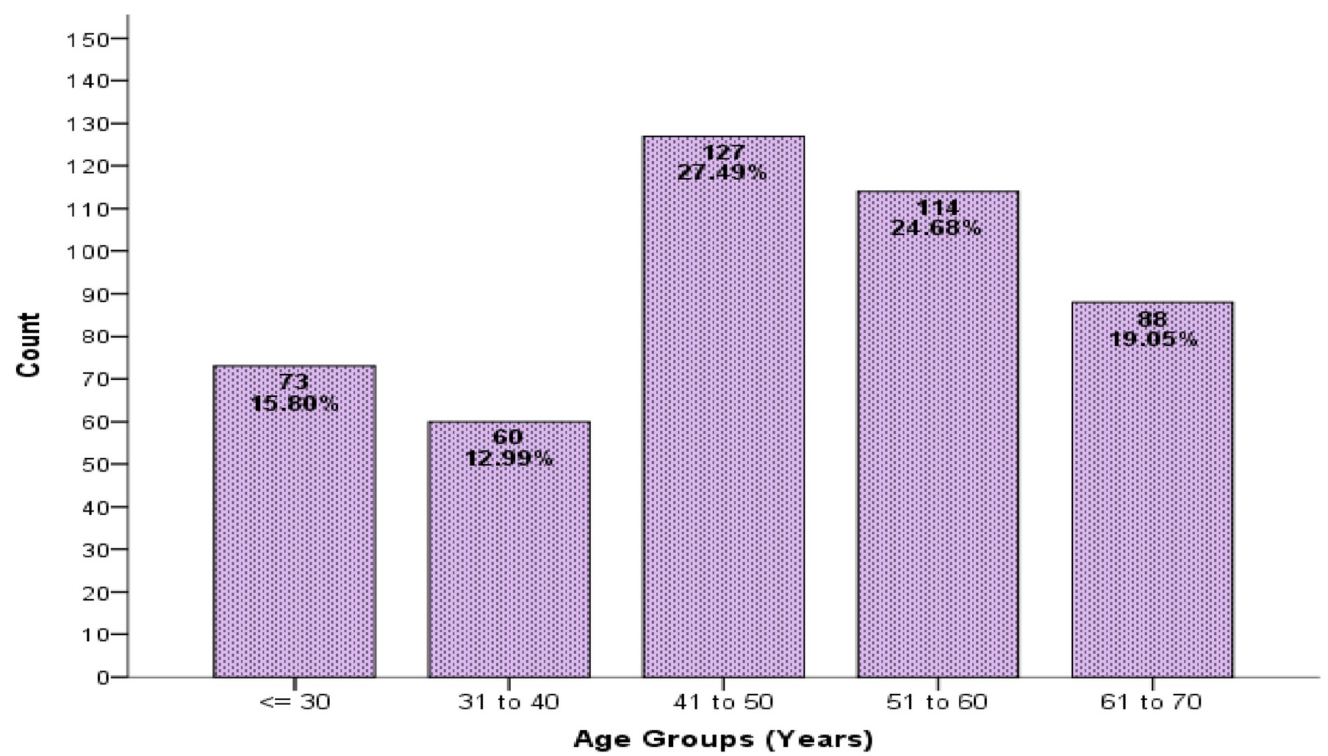

\section{FIGURE 3: Age distribution of patients}




\section{Cureus}

The mean age of the patients was $47.39 \pm 13.54$ years; similarly, the mean size of the abscesses was $4.99 \pm 2.26 \mathrm{~cm}$. There were $58.66 \%$ male and $41.34 \%$ female.

Histopathology confirmed the diagnosis of an intra-cerebral abscess in $42.2 \%(195 / 462)$ cases while echo-planar diffusion-weighted MRI findings showed only 38.5\% (178/462) cases had an intra-cerebral abscess. Regarding the false echo-planar diffusion-weighted MRI, 11 of 178 cases were false positive and 28 of 284 were diagnosed as false negative finding by echo-planar diffusion-weighted MRI. The sensitivity, specificity, PPV, NPV, and diagnostic accuracy of echo-planar diffusion-weighted MRI in the diagnosis of intra-cerebral abscesses was 85.64\%, 95.88\%, 93.82\%, 90.14\%, and 91.56\%, respectively. Kappa statistics ( $k=0.83)$ also showed excellent agreement between findings. A 95\% confidence interval was also reported for each statistics (Table 1).

\begin{tabular}{|c|c|c|}
\hline Parameter & Estimate & Lower-Upper (95\% Cls) \\
\hline Sensitivity & $85.64 \%$ & $(80.03,89.88)$ \\
\hline Specificity & $95.88 \%$ & $(92.77,97.68)$ \\
\hline Positive Predictive Value & $93.82 \%$ & $(89.27,96.51)$ \\
\hline Negative Predictive Value & $90.14 \%$ & $(86.12,93.09)$ \\
\hline Diagnostic Accuracy & $91.56 \%$ & $(88.67,93.76)$ \\
\hline Kappa Value & 0.83 & $(0.734-0.91)$ \\
\hline
\end{tabular}

TABLE 1: Diagnostic accuracy of echo-planar diffusion-weighted imaging in the diagnosis of intra-cerebral abscesses by taking histopathological findings as the gold standard

A stratification analysis was performed and observed that the diagnostic accuracy of echoplanar diffusion-weighted MRI in the diagnosis of intra-cerebral abscesses and Kappa statistics was high in all age groups and both sizes of abscesses ( $\leqslant 5 \mathrm{~cm},>5 \mathrm{~cm}$ ) (Tables 2-5). 


\section{Cureus}

\begin{tabular}{|l|l|l|}
\hline Parameter & Estimate & Lower-Upper (95\% Cls) \\
\hline Sensitivity & $90.98 \%$ & $(84.57,94.89)$ \\
Specificity & $97.1 \%$ & $(92.78,98.87)$ \\
Positive Predictive Value & $96.52 \%$ & $(91.4,98.64)$ \\
Negative Predictive Value & $92.41 \%$ & $(86.93,95.71)$ \\
Diagnostic Accuracy & $94.23 \%$ & $(90.7,96.47)$ \\
Kappa Value & 0.88 & $(0.76-1.0)$
\end{tabular}

TABLE 2: Diagnostic accuracy of echo-planar diffusion-weighted imaging in the diagnosis of an intra-cerebral abscess for below and equal to 50 years of age

\begin{tabular}{|c|c|c|}
\hline Parameter & Estimate & Lower-Upper (95\% Cls) \\
\hline Sensitivity & $76.71 \%$ & $(65.83,84.92)$ \\
\hline Speciticity & $94.57 \%$ & $(89.22,97.35)$ \\
\hline Positıve Predictive Value & $88.89 \%$ & $(78.8,94.51)$ \\
\hline Negative Predictive Value & $87.77 \%$ & $(81.29,92.22)$ \\
\hline Diagnostic Accuracy & $88.12 \%$ & $(82.93,91.88)$ \\
\hline Kappa Value & 0.1341 & $(0.59-0.87)$ \\
\hline
\end{tabular}

TABLE 3: Diagnostic accuracy of echo-planar diffusion-weighted imaging in the diagnosis of an intra-cerebral abscess for above 50 years of age 


\title{
Cureus
}

\begin{tabular}{|c|c|c|}
\hline Parameter & Estimate & Lower-Upper (95\% Cls) \\
\hline Sensitivity & $81.36 \%$ & $(73.38,87.35)$ \\
\hline Specificity & $97.35 \%$ & $(93.39,98.97)$ \\
\hline Positive Predictive Value & $96 \%$ & $(90.16,98.43)$ \\
\hline Negative Predictive Value & $86.98 \%$ & $(81.08,91.24)$ \\
\hline Diagnostic Accuracy & $90.33 \%$ & $(86.21,93.32)$ \\
\hline Kappa Value & 0.80 & $(0.68-0.91)$ \\
\hline
\end{tabular}

TABLE 4: Diagnostic accuracy of echo-planar diffusion-weighted imaging in the diagnosis of intra-cerebral abscesses that are $\leq 5 \mathrm{~cm}$ in size

\begin{tabular}{|c|c|c|}
\hline Parameter & Estimate & Lower-Upper (95\% Cls) \\
\hline Sensitivity & $92.21 \%$ & $(84.02,96.38)$ \\
\hline Specificity & $93.97 \%$ & $(88.07,97.05)$ \\
\hline Positive Predictive Value & $91.03 \%$ & $(82.62,95.58)$ \\
\hline Negative Predictive Value & $94.78 \%$ & $(89.08,97.59)$ \\
\hline Diagnostic Accuracy & $93.26 \%$ & $(88.82,96.02)$ \\
\hline Kappa Value & 0.85 & $(0.71-1.0)$ \\
\hline
\end{tabular}

TABLE 5: Diagnostic accuracy of echo-planar diffusion-weighted imaging in the diagnosis of intra-cerebral abscesses that are $>5 \mathrm{~cm}$ in size

\section{Discussion}

\begin{abstract}
A brain abscess remains a diagnostic challenge to clinicians and radiologists because the presenting clinical manifestations and neuroradiologic appearances are often nonspecific. Only $40 \%-50 \%$ of patients are febrile on examination. The more common signs and symptoms are those of any expanding intracranial mass: headache, altered mental status, focal sensorimotor deficits, seizure, nausea, and vomiting [10]. For this reason, a radiological diagnosis has particular importance [11]. Fever is characteristic of the invasion period of an abscess, whereas its encapsulation results in the normalization of body temperature; hence, the diagnosis depends on radiological investigations [11]. Sometimes, CT and conventional MRI cannot easily differentiate cystic and necrotic brain tumors from abscesses. Ebisu et al. were the first to describe the role of DWI here. The authors reported that abscesses appeared hyperintense on DWI and hypointense on apparent diffusion coefficient (ADC) mapping, whereas tumors appeared hypointense on DWI and hyperintense on ADC mapping [12]. Diffusion-weighted imaging is more practical in clinical use. It is a fast echo planar imaging technique and requires
\end{abstract}


less imaging time [13]. CT and MR imaging has high sensitivity for diagnosing ring-enhancing brain lesions but most of the time, it is difficult to differentiate between a neoplastic mass lesion and a brain abscess. DWI shows much promise in differentiating the differentials of ring-enhancing lesions [14].

A total of 462 patients, who were referred for MRI brain, presenting with a clinical suspicion of an intra-cerebral abscess on the basis of clinical signs and symptoms, were included. There were $58.66 \%$ male and $41.34 \%$ female in our study; this is in agreement with most series where males are affected more frequently than females [15-16]. We found above 40 years of age were more reflected and the mean age of the patients was $47.39 \pm 13.54$ years. Roche et al. in 2003 and Kastenbauer et al. in 2004 reported that a brain abscess is most common in the third decade of life but may occur at any age [16-17]. Abscesses due to para-nasal infections are most common between the ages of 10 and 30 years. Otogenic abscesses are most common in childhood and after 40 years of age.

Previous studies described a high accuracy of DWI in differentiating brain abscess from cystic/necrotic tumors. The differentials of ring-enhancing lesions include high-grade gliomas, metastases, abscesses, demyelinating diseases, and resolving hematomas [18]. There are reports that diffusion restriction can be seen in neoplastic cystic lesions. Similarly, increased diffusion and $\mathrm{ADC}$ value have been described, thus raising the question mark over the accuracy of this technique [19-20]. The sensitivity, specificity, PPV, NPV, and diagnostic accuracy of echoplanar diffusion-weighted MRI in the diagnosis of intra-cerebral abscesses was 85.64\%, 95.88\%, 93.82\%, 90.14\%, and 91.56\%, respectively. Kappa statistics ( $\mathrm{k}=0.83)$ also showed excellent agreement between findings. NInety-five percent confidence was also reported for each statistic. This study showed high sensitivity, specificity, positive, negative predictive value, and accuracy, which is in agreement with other studies. Lai et al. reported that DWI yielded a sensitivity of $93.33 \%$, a specificity of $90.91 \%$, a PPV of $93.33 \%$, and an NPV of $90.91 \%$ [21], and Reddy et al. reported the sensitivity of DWI for the differentiation of brain abscesses from nonabscesses was 96\%; specificity, 96\%; positive predictive value, 98\%; negative predictive value, 92\%; and accuracy was 96\% [9].

\section{Conclusions}

Brain abscesses and necrotic tumors are, most of the time, difficult to differentiate on routine conventional imaging, and prompt diagnosis is important, as an untreated brain abscess could be lethal. Diffusion imaging can aid in the diagnosis and further management plan so as to help in improved patient care. Although this sequence has high sensitivity and specificity, it should be used in addition to conventional imaging and not as a replacement for histopathology.

\section{Additional Information \\ Disclosures}

Human subjects: Consent was obtained by all participants in this study. Animal subjects: All authors have confirmed that this study did not involve animal subjects or tissue. Conflicts of interest: In compliance with the ICMJE uniform disclosure form, all authors declare the following: Payment/services info: All authors have declared that no financial support was received from any organization for the submitted work. Financial relationships: All authors have declared that they have no financial relationships at present or within the previous three years with any organizations that might have an interest in the submitted work. Other relationships: All authors have declared that there are no other relationships or activities that could appear to have influenced the submitted work.

\section{References}


1. Besharat M, Abbasi F: Brain abscess; epidemiology, clinical manifestations and management: a retrospective 5-year study. Iranian J Clin Infect Dis. 2010, 5:231-234.

2. Miranda HA, Castellar-Leones SM, Elzain MA, Moscote-Salazar LR: Brain abscess: current management. J Neurosci Rural Pract. 2013, 4:67-81. 10.4103/0976-3147.116472

3. Brouwer MC, Coutinho JM, van de Beek D: Clinical characteristics and outcome of brain abscess: systematic review and meta-analysis. Neurology. 2014, 82:806-813.

10.1212/WNL.0000000000000172

4. Tomar V, Yadav A, Rathore RK, et al.: Apparent diffusion coefficient with higher b-value correlates better with viable cell count quantified from the cavity of brain abscess. AJNR Am J Neuroradiol. 2011, 32:2120-2125. 10.3174/ajnr.A2674

5. Manzar N, Manzar B, Kumar R, Bari ME: The study of etiologic and demographic characteristics of intracranial brain abscess: a consecutive case series study from Pakistan. World Neurosurg. 2011, 76:195-200. 10.1016/j.wneu.2011.02.011

6. Honda H, Warren DK: Central nervous system infections: meningitis and brain abscess . Infect Dis Clin North Am. 2009, 23:609-623. 10.1016/j.idc.2009.04.009

7. Chiang IC, Hsieh TJ, Chiu ML, Liu GC, Kuo YT, Lin WC: Distinction between pyogenic brain abscess and necrotic brain tumour using 3-Tesla MR spectroscopy, diffusion and perfusion imaging. Br J Radiol. 2009, 82:813-820. 10.1259/bjr/90100265

8. Alam MS, Sajjad Z, Azeemuddin M, Khan ZA, Mubarak F, Akhtar W: Diffusion weighted MR imaging of ring enhancing brain lesions. J Coll Physicians Surg Pak. 2012, 22:428-431.

9. Reddy JS, Mishra AM, Behari S, Husain M, Gupta V, Rastogi M, Gupta RK: The role of diffusionweighted imaging in the differential diagnosis of intracranial cystic mass lesions: a report of 147 lesions. Surg Neurol. 2006, 66:246-250.

10. Whitley RJ, Schlitt M: Encephalitis caused by herpesviruses, including B virus . Infections of the Central Nervous System. Raven Press, New York; 1991. 41-59.

11. Rana S, Albayram S, Lin DD, Yousem DM: Diffusion-weighted imaging and apparent diffusion coefficient maps in a case of intracerebral abscess with ventricular extension. AJNR Am J Neuroradiol. 2002, 23:109-112.

12. Ebisu T, Tanaka C, Umeda M, et al.: Discrimination of brain abscess from necrotic or cystic tumours by diffusion weighted imaging. J Magn Reson Imaging. 1996, 14:1113-1136. 10.1016/S0730-725X(96)00237-8

13. Schaefer PW, Grant PE, Gonzalez RG: Diffusion-weighted. MR imaging of the brain . Radiology. 2000, 217:331-345. 10.1148/radiology.217.2.r00nv24331

14. Stadnik TW, Chaskis S, Michotte A, et al.: Diffusion wt. MR imaging of intracerebral masses: comparison with conventional MR imaging and histologic findings. AJNR Am J Neuroradiol. 2001, 22:969-976.

15. Chun CH, Johnson JD, Hofsteter M, Raff MJ: Brain abscess. A study of 45 consecutive cases . Medicine. 1986, 65:415-431.

16. Scheld MW, Whitley RJ, Marra CM: Infections of the Central Nervous System . Lippincott Williams \& Wilkins, Philadelphia; 2014.

17. Roche M, Humphreys H, Smyth E, et al.: A twelve-year review of central nervous system bacterial abscesses; presentation and aetiology. Clin Microbiol Infect. 2003, 9:803-809. 10.1046/j.1469-0691.2003.00651.x

18. Shetty G, Avabratha KS, Rai BS: Ring-enhancing lesions in the brain: a diagnostic dilemma . Iran J Child Neurol. 2014, 8:61-64.

19. Cartes-Zumelzu FW, Stavrou I, Castillo M, Eisenhuber E, Knosp E, Thurnher MM: Diffusionweighted imaging in the assessment of brain abscesses therapy. Am J Neuroradiol. 2004, 25:1310-1317.

20. Garg M, Gupta RK, Husain M, et al.: Brain abscesses: etiologic categorization with in vivo proton MR spectroscopy. Radiology. 2004, 230:519-527. 10.1148/radiol.2302021317

21. Chang SC, Lai PH, Chen WL, et al.: Diffusion-weighted MRI features of brain abscess and cystic or necrotic brain tumors: comparison with conventional MRI. Clin Imag. 2002, 26:227236. 10.1016/S0899-7071(02)00436-9 